\title{
Estimation of the time-dependent radioactive source-term from the Fukushima nuclear power plant accident using atmospheric transport modelling
}

\author{
Michael Schöppner ${ }^{\mathrm{a}, \mathrm{b}}$, Wolfango Plastino ${ }^{\mathrm{a}, \mathrm{b}, *}$, Pavel P. Povinec ${ }^{\mathrm{c}}$, Gerhard Wotawa ${ }^{\mathrm{d}}$, Francesco Bella ${ }^{\mathrm{a}}$, \\ Antonio Budano $^{\mathrm{b}}$, Mario De Vincenzi ${ }^{\mathrm{a}, \mathrm{b}}$, Federico Ruggieri ${ }^{\mathrm{b}}$ \\ ${ }^{a}$ University of Roma Tre, Department of Physics, Via della Vasca Navale 84, I-00146 Roma, Italy \\ ${ }^{\mathrm{b}}$ INFN, Section of Roma Tre, Via della Vasca Navale 84, I-00146 Roma, Italy \\ ${ }^{c}$ Comenius University, Department of Nuclear Physics and Biophysics, Mlynska dolina F-1, SK-84248, Bratislava, Slovakia \\ ${ }^{\mathrm{d}}$ Central Institute for Meteorology and Geodynamics, Hohe Warte 38, A-1190 Vienna, Austria
}

\section{A R T I C L E I N F O}

\section{Article history:}

Received 10 August 2011

Received in revised form

19 October 2011

Accepted 10 November 2011

Available online 3 December 2011

\section{Keywords:}

Fukushima accident

Atmospheric transport modelling

Caesium-137

Iodine-131

Source-term

\begin{abstract}
A B S T R A C T
Caesium-137 and Iodine-131 radionuclides released after the Fukushima Dai-ichi nuclear power plant accident in March 2011 were detected at monitoring stations throughout the world. Using the CTBT radionuclide data and the assumption that the Fukushima accident was the only source of these radionuclides, it was possible to estimate their time-dependent source-term fourteen days following the accident by using atmospheric transport modelling. A reasonable agreement was obtained between the modelling results and the estimated radionuclide release rates from the Fukushima accident.
\end{abstract}

(C) 2011 Elsevier Ltd. All rights reserved.

\section{Introduction}

Following the Tōhoku earthquake and the resulting tsunami on 11 March 2011 several reactors within the Fukushima Dai-ichi nuclear power plant (FD-NPP) were severely damaged. According to estimations published by the operator TEPCO (Tokyo Electric Power Company), the first radionuclides were detected on-site on 12 March 2011; the series of events with radionuclide releases can be reviewed on the International Atomic Energy Agency website (IAEA, 2011).

In order to assess an impact of the Fukushima accident on regional and global environment it is of high interest to determine radionuclide release rates from the FD-NPP site. A possible off-site method could be based on the International Monitoring System (IMS) currently installed by the Preparatory Commission for the Comprehensive Nuclear-Test Ban Treaty Organization (CTBTO). The IMS was designed for searching for undeclared nuclear activities such as nuclear weapons tests, but it can also be used as an effective monitoring system for tracing radionuclides released to the

\footnotetext{
* Corresponding author. University of Roma Tre, Department of Physics, Via della Vasca Navale 84, I-00146 Roma, Italy. Tel.: +39 0657337277; fax: +390657337102 .

E-mail address: plastino@fis.uniroma3.it (W. Plastino).
}

atmosphere from different sources. The IMS with a global network of sampling stations provides radionuclide data which can also be used for studying atmospheric processes (ZAMG, 2011). In this paper, however, the IMS data is used in a different way - as an inversion problem to reconstruct the Fukushima radionuclide source-term on the basis of world-wide atmospheric radionuclide measurements.

In the modelling exercise we have used three IMS stations: the closest station to the FD-NPP is RN38 station located at Takasaki/ Gunma, south-west of Tokyo, Japan; RN79 station located on Hawaii, USA, and RN70 station located in Sacramento (California, USA).

The atmospheric transport modelling was carried out using the GRID computing facility of the Italian National Institute of Nuclear Physics (INFN) at the University of Roma Tre. Preliminary results were reported at the 7th International Conference on Natural Computation (Plastino et al., 2011).

\section{Modelling background}

\subsection{Atmospheric transport modelling}

Particles, which are emitted in a certain time interval from a given point on the globe, are transported through the 


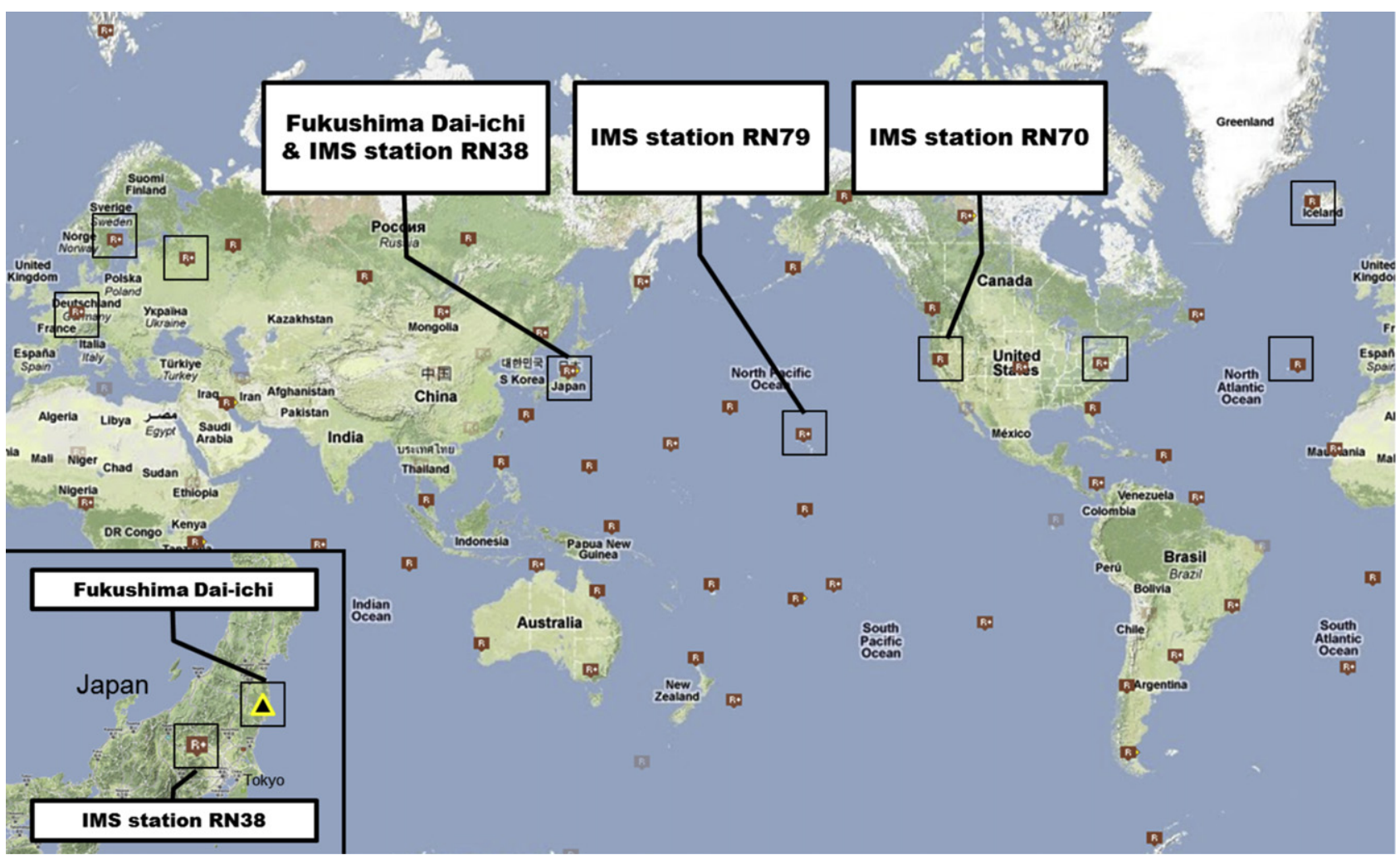

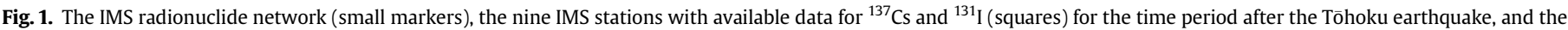

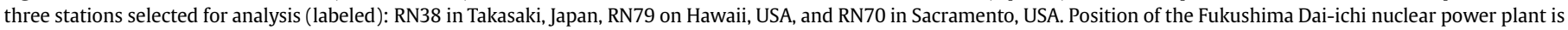
also shown.

atmosphere, and will arrive in a diluted concentration at certain other locations. Atmospheric Transport Modelling (ATM) can be used as a tool to estimate the time-dependent relation between two locations on a global (or regional) grid. These two points shall be called source and receptor, and the relation between them source-receptor-sensitivity (SRS). If now the emission of a source or the concentration at the receptor is known, the other parameter can be estimated via ATM. Of course, the results strongly depend on the meteorological conditions during the transport period, as well as on local atmospheric patterns that are not resolved by the simulation, which can lead to altered signals (Plastino et al., 2010). It is important to keep in mind that particles emitted in one certain time interval can contribute in multiple times and at different arrival times to the concentration at the receptor site via various trajectories through the atmosphere.

The relation between a source, which emits particles into the atmosphere, and the concentration at a receptor can be described with a source-receptor sensitivity matrix. The concentration $c(B q /$ $\mathrm{m}^{3}$ ) at any given receptor can be expressed as the product of a spatio-temporal source field $S(\mathrm{~Bq})$ and a corresponding sourcereceptor sensitivity field $M\left(\mathrm{~m}^{-3}\right)$ at discrete locations $(i, j)$ and time intervals $n$ :

$c=M_{i j n} \cdot S_{i j n}$

The field $S$ is a multidimensional array of sources, which is transformed by the multidimensional array of multiplicators $M$ into the concentration $c$ that is measured at the receptor (Wotawa et al., 2003). Here $M$ presents the sensitivity between source and receptor, whereas the inverse element of $M$ can be depicted as a dilution volume. However, while the underlying calculations are naturally three-dimensional, the produced SRS matrix $M$ is only two-dimensional.

The ATM software can simulate the transport of particles released from point, line, area or volume sources. The simulations can include long-range and mesoscale transport, diffusion, dry and wet deposition, as well as radioactive decay into the calculations. This means that the radioactive decay can be simulated with the same time resolution as the output frequency of the atmospheric transport modelling itself. In ATM it is usually distinguished between a forward and backward modelling, where both methods have advantages and disadvantages. Forward modelling is usually more efficient when the number of known sources is limited and the receptors are undefined. On the other hand backward modeling is usually more efficient when the number of receptors is limited and the sources are unknown. ATM was proven to be a valid tool for determining the SRS matrices (Wotawa et al., 2003).

In the case of the FD-NPP accident and the following IMS measurements, both the source and receptor locations are known. Therefore, both possibilities could be used in this case to determine the sensitivity between the source and the receptor. The results presented in this paper are based on the backward mode. In the backward mode the source-receptor sensitivity $M\left(\mathrm{~m}^{-3}\right)$ is related to the adjoint concentration output $C\left(\mathrm{~Bq} / \mathrm{m}^{3}\right)$ of the model:

$M_{i j n}=C_{i j n} / A$

where $A$ is the total (adjoint) activity released in the backward run (Wotawa et al., 2003; Stohl et al., 2011). The assumed source duration is equal to the time resolution of the model output. A longer acting source can be included by adding SRS elements from neighboring time intervals. 


\subsection{Data sources}

The German Federal Office for Radiation Protection (BfS) has published the ${ }^{137} \mathrm{Cs}$ and ${ }^{131}$ I concentrations at selected IMS stations for the time period following the FD-NPP accident (BfS, 2011). Due to the general west to east wind direction in the northern hemisphere, the following three IMS stations have been selected for this comparison: RN38 in Takasaki/Gunma, Japan (250 km distance to the FD-NPP; RN79 on Hawaii, USA (6200 km); and RN70 in Sacramento, USA (8800 km) (Fig. 1).

\subsection{Software and parallel computing}

The FLEXPART software package (Stohl et al., 2011) based on the Lagrangian model was used for the ATM calculations presented in this paper. The CTBTO also utilized this package to monitor emissions from nuclear weapon tests (Wotawa et al., 2003; Zähringer et al., 2009; Plastino et al., 2010). It is desirable to run multiple FLEXPART jobs in order to calculate SRS fields for a number of different sources and receptors. Since the FLEXPART source code is highly linear, it is not efficient to parallelize it. Nevertheless, a userdefined number of FLEXPART runs can be sent to a cluster (or grid) computer for parallel execution. FLEXPART was ported to a local cluster computer at the INFN and the Department of Physics of the University of Roma Tre Grid in order to be able to do multiple parallel runs with minimum time delay (Plastino et al., 2011). The
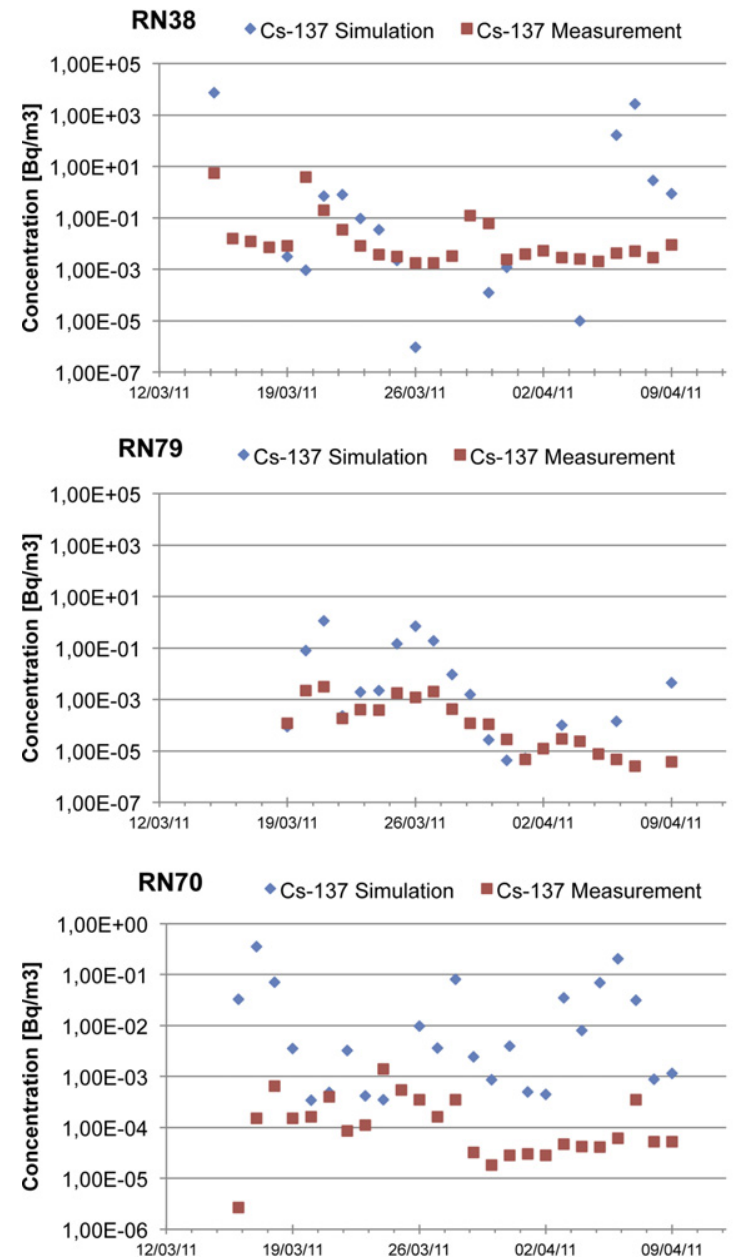

porting on the Grid Infrastructure is in progress and will allow running the program on the geographically distributed nodes of the EUMEDGrid-Support Grid Infrastructure.

The simulations were carried out for particles of ${ }^{137} \mathrm{Cs}$ and ${ }^{131} \mathrm{I}$. Distinguishing between both isotopes allows taking into account different loss processes during the radionuclide transport. The simulated loss processes are including radioactive decay as well as dry and wet deposition.

\subsection{Geographical setting}

The FLEXPART simulations have been accomplished in the backward mode and with $1^{\circ} \times 1^{\circ}$ resolution (latitude $\times$ longitude) for the IMS stations RN38 in Takasaki, Japan, RN79 on Hawaii, USA, and RN70 in Sacramento, USA, during the first 30 days after the first on-site detection of radionuclides.

\subsection{Radionuclide release-rate scenarios}

A worst case scenario of the emission of $10^{19} \mathrm{~Bq} /$ day for both ${ }^{137} \mathrm{Cs}$ and ${ }^{131} \mathrm{I}$ (constant release on the 12 March 2011) was used and the resulting estimations were compared with real measurements. First estimations of worst case emissions served as input data for atmospheric simulations. These estimations were then compared with the actual IMS measurements. Further, an approach is presented to determine the time dependent source term for ${ }^{137} \mathrm{Cs}$ and
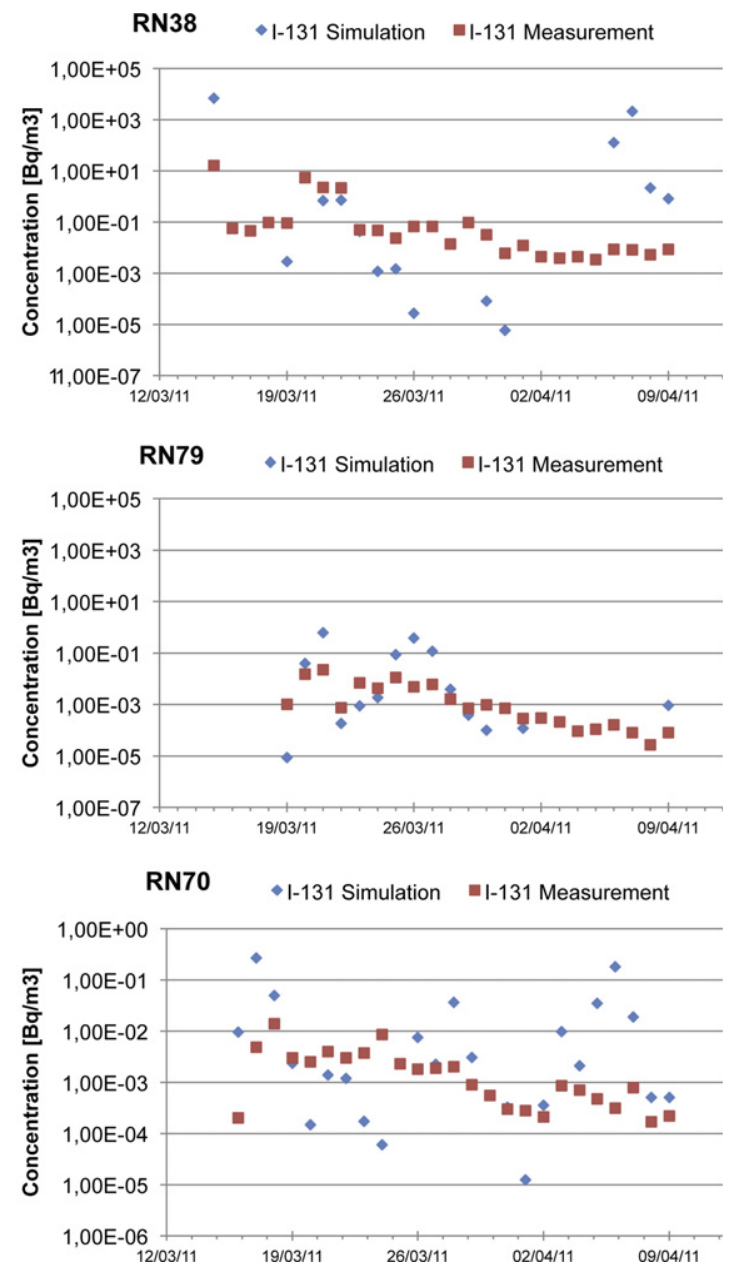

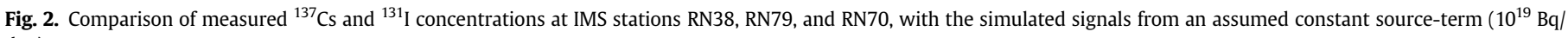
day). 
${ }^{131}$ I at the FD-NPP. The resulting time dependent source term is compared with the on-site events of the first two weeks after the release of radionuclides, as far as these are publicly available.

\section{Results and discussion}

\subsection{Worst case scenario}

The calculated and measured results are compared in Fig. 2. As can be seen the three comparisons between the estimations and measurements are diverse and vary between the stations as well as between the radionuclides. In the case of ${ }^{137} \mathrm{Cs}$, the simulation for the Japanese station RN38 depicts some significant characteristics of the measurement. However, for some sampling days the estimations are clearly too high, while for other days clearly too low. For the second closest station, RN79 on Hawaii, the general outline of the signal is picked up well by the simulation; however, the simulation tends to produce rather high estimations compared to the measurements. The third station, RN70 in California, has measured concentration quite below the estimations. On the other hand, the comparisons in the case of ${ }^{131}$ I iodine show that the estimation of a constant $10^{19} \mathrm{~Bq} /$ day source term produces estimations, which are on average similar to the measurements.

The reasons for the divergence between simulations and measurements can be manifold. The fact that the two remote stations, RN79 and RN70, suggest a lower source term than the worst case estimation, where the closest station, RN38, does not hint to unaccounted regional meteorological conditions. In the case of the FD-NPP and the Japanese IMS station the distance between source and receptor is only about $250 \mathrm{~km}$, which puts them on neighbouring points of the global $1^{\circ} \times 1^{\circ}$ grid. Therefore, local weather patterns, which are not resolved by the simulation software can be responsible for the transport of higher amounts of airborne material from the source to the receptor (Plastino et al., 2010). Naturally, this would result in measuring a concentration which is higher than the estimated one, even if the estimation would be based on a real source term, which has not been constant in time.

It can be concluded from the other two contemplated stations that the real source-term at the FD-NPP emitted fewer particles in the case of ${ }^{137} \mathrm{Cs}$ than assumed in the worst case scenario. Based on these results a source term of $10^{17}-10^{18} \mathrm{~Bq} /$ day seems reasonable. For ${ }^{131} \mathrm{I}$, however, these first results suggest a source term not too different from the worst case scenario of $10^{19} \mathrm{~Bq} /$ day.

\subsection{Time-dependent source-term}

Firstly, one has to distinguish between the release date and the detection date, and furthermore remember that emissions from the same release day can be detected at multiple detection dates. Viceversa, the concentration that was measured on one detection day is likely to be a combination of different release days. Since the experimental data from the IMS stations (BfS, 2011) have a time resolution of one day, the same time resolution was chosen for the source-term. In the case of one receptor and one source, each detection date is characterized by its relationship to all possible release dates by the SRS matrix, which determines the dilution factors. This means that the SRS matrix of each detection day can be split into different contributions from possible release dates.

Now it is necessary to adjust the source term for each release day in a way to minimize the differences between the simulation and the experimental measurement. In the frame of this study this was done in order to test the applicability of this approach. Naturally this is a highly recursive process of step by step optimization of the single source-terms of each day. However, it is beneficial to start

with detection dates, which according to the transport model are only affected by one release date. After adjusting these sourceterms it is possible to work on the detection dates, which have various contributions from different release days. Of course, this is affecting already adjusted values, so it might also be necessary to readjust the already established source-terms.

The adjusted source-terms for the first two weeks after the earthquake are presented together for the three IMS stations, but separately for the two radionuclides in Fig. 3. This means that the measurements of each station were used to develop their respective time dependent source-terms. The radionuclide data show that the time dependent source-terms predicted by the measurements at three different stations were not congruent for both radionuclides. Contemplating one radionuclide and three stations, peaks and other characteristics of the time series appear at different dates. On the other hand, when comparing both radionuclide source-terms at one single station, a general compliance in the development of the time series can be observed.

The main point is, of course, to compare these results with the time-line of events at the FD-NPP. Multiple explosions took place on 12,14 and 15 March (IAEA, 2011). On 18 March high amounts of smoke was observed; with a decreasing quantity in the following days. However, published data from TEPCO (GRS, 2011) report particularly high peaks in the on-site dose rate $(\mathrm{mSv} / \mathrm{h})$ for 15 and 16 March. Some of these event reports are depicted in the estimated source-terms, e.g. the general increase of emissions for 18 and 19 March. On the other hand, for 15 and 16 March, when high dose rates on site were reported, the source-terms show no

\section{Caesium-137}
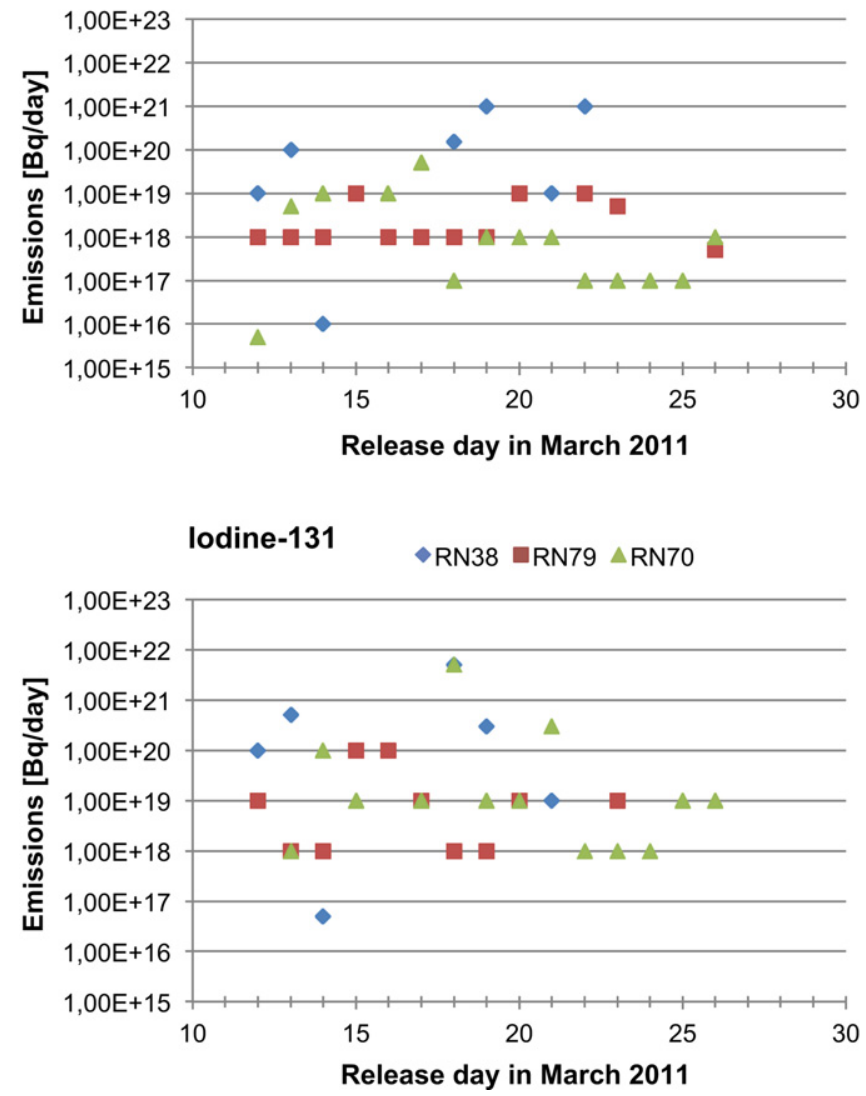

Fig. 3. Estimation of a time dependent source-term for ${ }^{137} \mathrm{Cs}$ and ${ }^{131} \mathrm{I}$ from the Fukushima Dai-ichi NPP. The estimations are based on the results from RN38, RN79, and RN70 stations. 
particular increase. Other characteristics of the estimated sourceterms seem to be off by one day. For example, on 13 March when explicitly no explosions occurred, the estimation from RN38 shows a peak which could also correspond to the explosions on 12 or 14 March. Effects like these could be explained by local meteorological patterns that would retain particles, and these are not resolved by the simulation.

The partial compliance of the results with the time-line of events shows a weakness of the ATM. It is natural that at certain times a meteorological link between the release and the detection is too weak to deduct a significant interpretation. At other times this link can produce decent results. By putting the source-terms from different stations in one diagram, each station can show the resolved peaks. An occurrence of low values or a plateau can be a sign for a weak meteorological link between the source and the receptor, while a peak should be a sign for resolved emissions. Here the combined results from different IMS stations can improve our understanding of the situation. Especially on regional scales, a smaller than the used $1^{\circ} \times 1^{\circ}$ resolution could produce better estimations than presented in this study.

\section{Conclusions}

The presented simulations with improved settings, i.e. dry and wet deposition, resulted in a better estimation of the source-term than previously reported (Plastino et al., 2011). The scenario assuming a worst case situation suggests generally higher sourceterms, which are in reasonable agreement with the reported values (IAEA, 2011). For ${ }^{137} \mathrm{Cs}$ emissions of $10^{17}-10^{18} \mathrm{~Bq} /$ day seem to be reasonable, while for ${ }^{131}$ I even continuous emissions of $10^{19} \mathrm{~Bq} /$ day could be possible.

The introduced approach to determine the time dependent emissions using the inversion problem approach led to an improved estimation of the source-terms. However, the presented source-terms do not perfectly describe the time-line of events when each radionuclide and station were considered separately. When they were considered together, it was possible to deduct a much clearer picture. As mentioned, this is most likely due to unavoidable weak links between certain release and detection times caused by meteorological conditions. Nevertheless, a network approach with multiple stations certainly improves the situation.

Future work in this field aims with usage of a least squares method, and furthermore an automated network analysis. This, of course, can also be useful to determine other radionuclide sources with the IMS network.

\section{Acknowledgments}

The authors highly acknowledge the support by the National Scientific Committee Technology of INFN for the ERMES project, and the European Commission under the FP7 programme for the EUMEDGRID project (Grant RI-246589). The authors are grateful to the Grid Lab of INFN and the Department of Physics of University of Roma Tre, and specifically to Federico Bitelli. PPP acknowledges a support provided by the EU Research and Development Operational Programme funded by the ERDF (project No. 26240220004).

\section{References}

BfS (German Federal Office for Radiation Protection), 2011. Spurenanalyse deutscher Messstellen und weiterer Spurenmessstellen weltweit. http://www.BfS.de/en/ ion/imis/spurenmessungen.html.

GRS (Gesellschaft für Anlagen und Reaktorsicherheit mbH), 2011. http://fukushima. grs.de/sites/default/files/Radiation-Data_Dai-ichi_20110319-1800.pdf.

IAEA (International Atomic Energy Agency), 2011. Fukushima Nuclear Accident Update. http://iaea.org/newscenter/news/tsunamiupdate01.html.

Plastino, W., Plenteda, R., Azzari, G., Becker, A., Saey, P.R... Wotawa, G., 2010. Radioxenon time series and meteorological pattern analysis for CTBT event categorisation. Pure and Applied Geophysics 167, 559-573.

Plastino, W., Schöppner, M., Povinec, P., Wotawa, G., Bella, F., Budano, A., De Vincenzi, M., Ruggieri, F., 2011. Atmospheric transport modeling based estimation of radioactive release from the Fukushima Dai-ichi nuclear power plant accident. In: Seventh International Conference on Natural Computation, vol. 4 IEEE, Shanghai, pp. 2027-2030.

Stohl, A., Sodemann, H., Eckhardt, S., Frank, A., Seibert, P., Wotawa, G., 2011. The Lagrangian Particle Dispersion Model FLEXPART, Version 8.2. Norwegian Institute of Air Research, Kjeller, Norway. http://transport.nilu.no/flexpart.

Wotawa, G., De Geer, L.E., Denier, P., Kalinowski, M., Toivonen, H., D’Amours, R, Desiato, F., Issartel, J.P., Langer, M., Seibert, P., Frank, A., Sloan, C., Yamazawa, Y., 2003. Atmospheric transport modelling in support of CTBT verification overview and basic concepts. Atmospheric Environment 37, 2529-2537.

Zähringer, M., Becker, A., Nikkinen, M., Saey, P., Wotawa, G., 2009. CTBT radioxenon monitoring for verification: today's challenges. Journal of Radioanalytical and Nuclear Chemistry 282, 737-742.

ZAMG (Central Institute for Meteorology and Geodynamics, Austria), 2011 http://www.zamg.ac.at/aktuell/index.php?seite=2. 